\title{
Grazynes: Carbon-Based Two-Dimensional Composites with Anisotropic Properties
}

\author{
Saeedeh Kamalinahad, a,b Francesc Viñes, a and Pablo Gamallo, \\ ${ }^{a}$ Departament de Ciència de Materials i Química Física \& Institut de Química Teòrica i \\ Computacional (IQTCUB), Universitat de Barcelona, c/ Martí i Franquès 1-11, 08028 \\ Barcelona, Spain; and ${ }^{\mathrm{b}}$ Department of Chemistry, Faculty of Science, Arak University, \\ 38156-8-8349 Arak, Iran.
}

*Corresponding author. E-mail: gamallo@ub.edu (Pablo Gamallo)

Tel. 0034934039308

ORCID IDs

Pablo Gamallo https://orcid.org/0000-0002-8531-8063

Francesc Viñes https://orcid.org/0000-0001-9987-8654 


\begin{abstract}
A new family of two-dimensional carbon allotropes is presented, based on graphene stripes linked to each other by acetylenic connections. The large amount of allowed connectivities demands for a family name to them: Grazynes. The present study reports the energetic, structural, elastic, and electronic physicochemical properties of a set of simple grazynes by means of density functional theory based calculations, suggesting also possible synthetic routes. The main results conclude that these are exotic yet stable materials, stiffer than graphene in the acetylenic direction, highly anisotropic, and with the presence of Dirac points in the reciprocal space along the graphene stripes direction resistant to strain, regardless of its direction. Thus, grazynes infer directionality in electron conductivity and resilience to the materials stretching/compression, quite important, for instance, in the nanoelectronics applicability point of view.
\end{abstract}




\section{INTRODUCTION}

The last decade has witnessed the bloom of C-based two-dimensional (2D) materials, ${ }^{1}$ mostly leaded by graphene, ${ }^{2}$ and posteriorly followed by other, more exotic $2 \mathrm{D}$ carbon allotropes, e.g., graphynes, ${ }^{3}$ graphane, ${ }^{4}$ and graphone, to name a few. ${ }^{5}$ The applied research on them has even opened the gate towards other few-layered non C-based materials. ${ }^{6}$ Focusing on the 2D Carbon allotropes group, they have become richer in their types and diversity, and the forecasted structures experimentally synthesized and/or isolated, spurred by theoretical predictions of their appealing properties; see e.g. the corroborated larger electrical conductivity of graphynes - 2D periodic arrangements of $\mathrm{C}$ atoms displaying $s p$ and $s p^{2}$ orbital hybridizations - compared to graphene, ${ }^{7}$ as previously predicted by computational simulations. ${ }^{3}$

From the nanoelectronics applicability point of view, it would be highly desirable to infer directionality in the electron conductivity of such 2D materials, a feature that can be externally inducible, but that can be intrinsically/structurally prompted as well, as does happen in anisotropic graphyne structures. ${ }^{3}$ Directionality may also affect other properties, such as compressibility, and this correspondence is de facto both ways, as compression/expansion of 2D lattices may affect the network structure and, in turn, its electronic structure, ${ }^{8}$ a critical point in many 2D C-based Covalent Organic Frameworks (COFs). ${ }^{9,10}$ Therefore, 2D carbon allotrope materials with inherent anisotropic properties are technologically desirable so as to control and/or exploit the elastic/electronic properties at will on a given preferred direction. Here we present a new family of $2 \mathrm{D}$ carbon allotropes that accomplish this task, to which we refer to as grazynes in the following. These allotropes can be also inherently tagged as graphynes, given that they contain both $s p^{2}$ and $s p \mathrm{C}$ atoms, although, at variance with graphynes, their directional display is inherently determined by the atomic 
hybridization, a point not achieved in graphynes. As observed in Figure 1, the $s p^{2}$ graphenic building blocks, regarded as graphene stripes, are concurrently aligned on a given direction, and transversally interlinked by acetylenic building blocks. The vast variety of graphene stripe widths and acetylenic lengths as well as the unit cell periodicity implies a rich palette of building blocks connectivity, allowing for such materials tailoring at will.

Figure 1. [n],[1]-grazyne structures with $n=1$ (left), 2 (middle), and 3 (right). Red lines fend the periodic unit cell. Note that the three structures correspond to variable widths of graphene stripes and the minimum length of acetylenic linkages $(m=1)$, the last ones occurring at each linking position, i.e., with $p_{l}=0$.

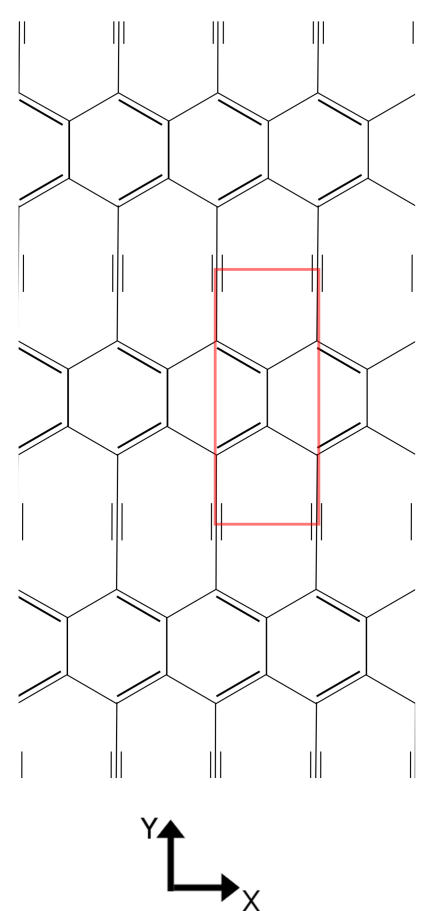

[1],[1]-grazyne

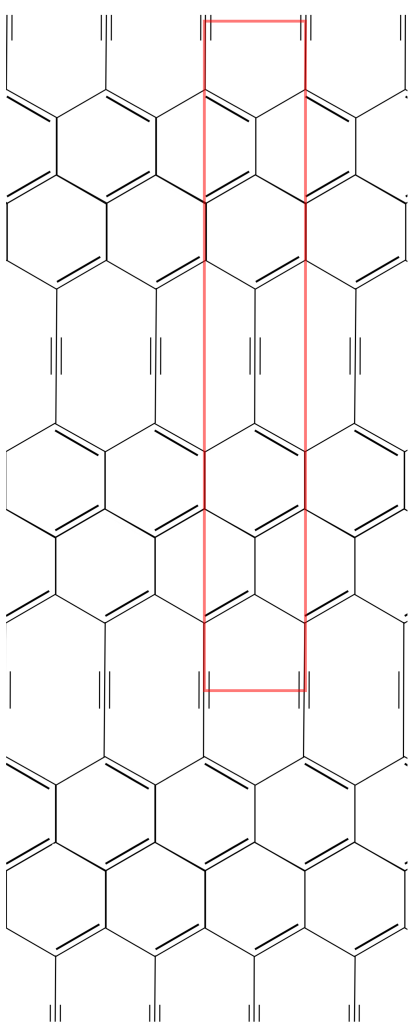

[2],[1]-grazyne

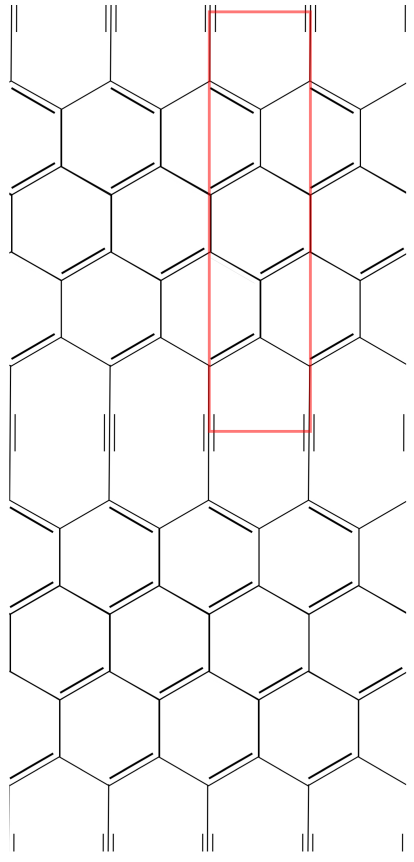

[3],[1]-grazyne 
Consequently, the grazyne structures —whose proposed name combines graphene with acetylenic linkages, with a sonority clear distinguishable from graphynes - can be catalogued according to the width of the graphene stripes, which can be of $[n]$-rings $(n=1,2,3, \ldots)$, and to the length of the acetylenic linkages, which can be of $[m]-\mathrm{C} \equiv \mathrm{C}$ units $(m=1,2,3, \ldots)$, along with their alternation in the unit cell $\{p\}$ indicating how many empty linking locations are needed until repetition (e.g., $[n],[m]\{p\}$-grazyne defines one grazyne with the same graphene stripes, acetylenic linkages, and $\left[n_{1}, n_{2}, \ldots\right],\left[m_{1}, m_{2}, \ldots\right]\left\{p_{1}, p_{2}, \ldots\right\}$-grazyne defines the alternation of $n_{1}$ and $n_{2}$ graphene stripes with $m_{1}$ and $m_{2}$ acetylenic lengths with alternation $p_{1}$ and $p_{2}$ ). Figure 1 shows the three grazyne structures studied in the present work (i.e., [1],[1]\{0\}grazyne, [2],[1]\{0\}-grazyne and [3],[1] \{0\}-grazyne). As can be seen in Figure 1, and for clarity purposes, when the acetylenic bridges occupy all the possible linking positions, the $\{0\}$ index can be omitted. Moreover, Figure 2 shows two grazyne structures, one with different alternating graphenic stripes (i.e., $[1,3],[1]\{0\}$-grazyne) and another where acetylenic linkers do not occupy all the possible linking points (i.e., $[1,3],[2]\{2\}$-grazyne) 
Figure 2. Example of $\left[n_{1}, n_{2}\right],\left[m_{1}, m_{2}\right]\left\{p_{1}, p_{2}\right\}$-grazyne structures with $n_{1}=1$ and $n_{2}=3$ with $m_{1}=1$ (left) and $m_{1}=2$ (right). The right structure exhibits $p_{1}=2$ alternation in the acetylenic linkages. Red lines fend the periodic unit cell.

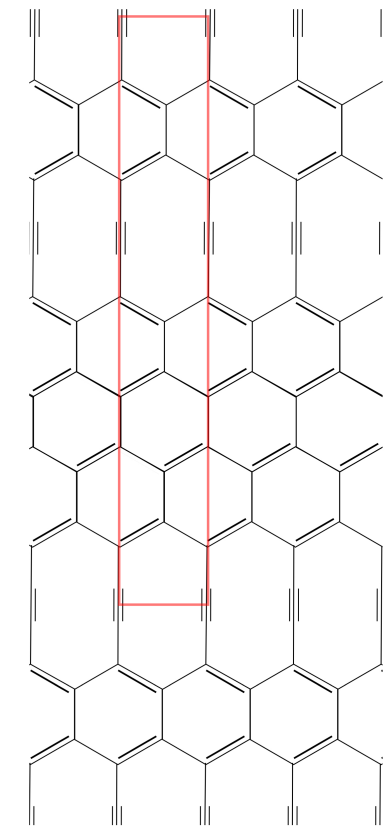

$[1,3],[1]$-grazyne

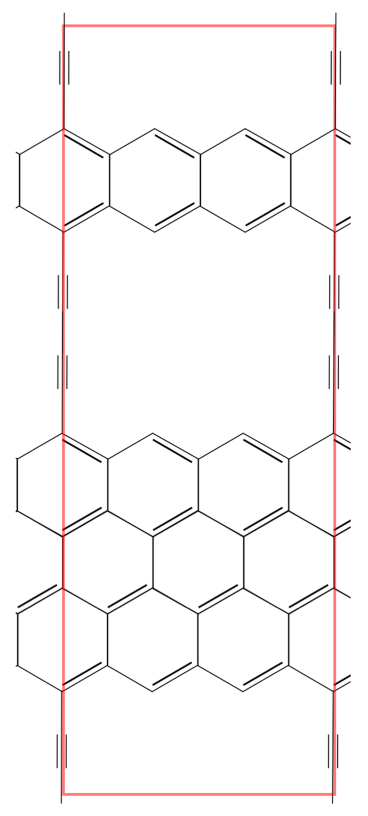

$[1,3],[2]\{2\}$-grazyne

\section{COMPUTATIONAL DETAILS}

Here we have analyzed, by means of first-principles calculations, the three simplest grazyne structures (i.e., [1],[1]-grazyne, [2],[1]-grazyne and [3],[1]-grazyne, see Figure 1) with different graphene stripe width. To this end Density Functional Theory (DFT) calculations have been performed, as done previously in the past for graphynes and COFs, ${ }^{3,9,10}$ exploiting the systems periodicity by imposing periodic boundary conditions. Two ab initio codes have been employed, the Vienna Ab initio Simulation Package (VASP) ${ }^{11}$ and the Fritz-Haber Institute Ab Initio Molecular Simulation (FHIAIMS) Package. ${ }^{12}$ 
Optimizations were carried out using VASP where valence electrons are explicitly treated, ${ }^{11}$ whereas core electrons are described by the Projector Augmented Wave (PAW) method. ${ }^{13}$ The kinetic energy cutoff for the plane-wave expansion of the valence electron density was set to 850,1200 , and $1050 \mathrm{eV}$ for [1],[1]-, [2],[1]-, and [3],[1]-grazynes, respectively. These values are large enough to obtain energies converged below $1 \mathrm{meV}$ per unit cell in all cases. Long-range dispersion corrections were included through the pairwise D2 force field as developed by Grimme, ${ }^{14}$ even though previous studies highlight the minimum impact that other corrections, such as D3, can have on 2D C-based materials, with variations of cell parameters below 0.0001 $\AA$, and changes in energy below $1 \mathrm{meV},{ }^{15,16}$ although one has to keep in mind that the use of one or another correction can sensibly influence the stacking of such sheets, or their interaction with other material surfaces. ${ }^{16}$ A vacuum layer of $20 \AA$ was added perpendicular to the materials layer direction to prevent any interaction between adjacent grazyne layers. All optimizations were carried out spin polarized, given that open-shell configurations are energetically reachable in carbon-based 2D Covalent Organic Radical Frameworks (CORFs), ${ }^{9,17}$ although these spin-polarized optimizations revealed either non magnetic moments or negligible magnetic moments in the studied cases, and so, all posterior single-point calculations were performed non spin-polarized to reduce the computational cost.

The systems were fully relaxed in the grazyne plane with an electronic energy convergence criterion of $10^{-5} \mathrm{eV}$ per unit cell and using a conjugated gradient method for the atomic relaxations until the Hellmann-Feynman forces acting on atoms became smaller than $0.01 \mathrm{eV} \cdot \AA^{-1}$. Moreover, the cell vectors containing the $2 \mathrm{D}$ structure were allowed to relax to accommodate the material. After the simultaneous optimization of atomic positions and cell vectors, the slight modifications of the vacuum region were reset to $20 \AA$, and ensuring afterwards that both the electronic and atomic convergence 
criteria were met by carrying out a single-point calculation. Test calculations with a stronger optimization criterion (i.e., of $0.001 \mathrm{eV} \cdot \AA^{-1}$ ) revealed negligible changes of only meV. The minimum character of the optimized structures was evaluated by vibrational frequency analyses of $0.03 \AA$, showing in all cases that the optimized structures correspond to true minima in the potential energy surface. Further than that, atomic out-of-plane displacement of carbon atoms and further relaxations were also tested, although the final structures back relaxed to the perfectly planar structures. Finally, layer rumplings and other collective deformations were applied and posteriorly relaxed, including graphenic or acetylenic rumplings, or both in opposite directions, similar to the observed deformations of graphyne upon molecular adsorption upon. ${ }^{18}$ In all cases the same back relaxation to the perfectly planar optimal structures was observed, confirming the stability of the structures.

During the electronic structure calculations, the reciprocal spaces were sampled using optimal $\Gamma$-centered Monkhorst-Pack ${ }^{19}$ k-point grids of $20 \times 20 \times 1,17 \times 13 \times 1$, and $19 \times 19 \times 1$ dimensions for [1],[1]-, [2],[1]-, and [3],[1]-grazynes, respectively, given that test calculations with lighter k-point meshes of $13 \times 9 \times 1,13 \times 3 \times 1$, and $13 \times 5 \times 1$ respectively, and a $750 \mathrm{eV}$ cutoff energy yielded converged cohesive energies but cell parameter variations of $\pm 0.002 \AA$ and spurious residual magnetic moments of 0.0342 , 0.0308 , and $-0.1231 \mu_{\mathrm{B}}$, respectively. The grazyne structures have been described within the Generalized Gradient Approximation (GGA) for the exchange-correlation functional, using for this purpose the functional developed by Perdew, Burke, and Ernzerhof (PBE), ${ }^{20}$ a functional proven to be accurate enough in the description of carbon-based materials. ${ }^{3,9}$ As far as strains along the grazyne sheet directions were concerned, the elastic properties were derived from energy calculations performed on 
the same unit cells depicted in Figure 1, where the $a$ and $b$ cell parameters correspond to those located on $x$ - and $y$-directions, respectively.

In order to obtain the systems band structures, the geometrical structures were optimized again using the seamless parallel FHI-AIMS package, ${ }^{12}$ where the electron density is described with a basis set of Numerical Atomic Orbitals (NAO). The PBE0 hybrid $^{21}$ functional has been used including an all electron description and taking into account scalar-relativistic effects at the Zero Order Regular Approximation (ZORA) level. ${ }^{22}$ A Tier-1 basis set together with light grid options have been chosen, which is of quality similar or even higher than Dunning ${ }^{23}$ GTO aug-cc-pVDZ. ${ }^{24}$ Note that the use of hybrid PBE0 instead of PBE-D2 prevents the unduly reduction of the materials bandgap, if any, as the self-interaction error gets counteracted by the Hartree-Fock exchange fraction present in PBE0. The band structures have been computed sampling energy bands in the $\Gamma$-X-S-Y quadrant of the bidimensional k-space, see Figure 3, evaluating all bands on 200 k-points of each sampled segment of the Brillouin zone. In the proximity of Dirac points, Fermi velocities were estimated from energy vs. reciprocal length slopes. ${ }^{9}$ In the case of band structures calculations, a tighter electronic energy convergence criterion was applied $\left(10^{-7} \mathrm{eV}\right)$.

Figure 3. Paths of the reciprocal k-space evaluated when gaining the grazynes band structures.

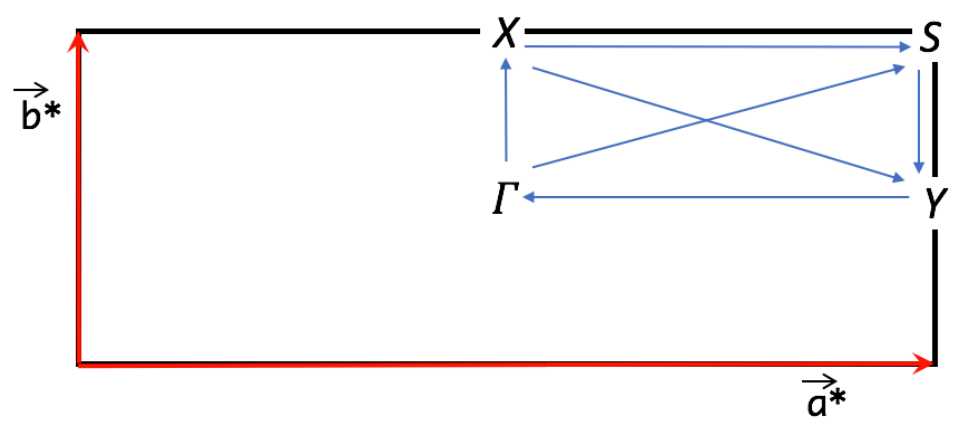


The grazynes energetic stabilities were analyzed by computing the cohesive energies once the in-plane cell parameters were optimized. These cohesive energies per C atom, $E_{c o h}$, were computed according to Equation (1), where $E_{G}$ is the total energy of the grazyne unit cell, $E_{C}$ is the total energy of a carbon atom in its vacuum ground electronic state (i.e., in its triplet ${ }^{3} \mathrm{P}$ ground state), and $n$ the number of carbon atoms per unit cell. According to this notation, the more positive the $E_{c o h}$, the higher the stability.

$$
E_{c o h}=\frac{n \cdot E_{C}-E_{G}}{n}
$$

The elastic properties of any $2 \mathrm{D}$ material in the linear regime can be described by two independent constants; the in-plane stiffness, $C$, that characterizes the rigidity or the flexibility of the material, and the Poisson's ratio, $v$, which defines the mechanical response to an external stress. Most of the materials tend to compress in one direction when they are expanded in a perpendicular one. This phenomenon, known as Poisson's effect, can be measured through the ratio of the transverse contraction strain to axial or longitudinal expansion strain, namely, the Poisson's ratio,

$$
v=-\frac{\varepsilon_{\text {trans }}}{\varepsilon_{\text {axial }}}
$$

These elastic constants can be deduced from DFT calculations through Equation (3), which relates the total energy of the system and the applied strain within the harmonic approximation, where $E$ is the energy of the system when the strain is applied, $E_{0}$ is the energy of the unstrained structure, and $\varepsilon_{x}$ and $\varepsilon_{y}$ are the applied strains along the parallel and perpendicular directions of the graphene stripes of grazynes, respectively.

$$
E=c_{1} \varepsilon_{x}^{2}+c_{2} \varepsilon_{y}^{2}+c_{3} \varepsilon_{x} \varepsilon_{y}+E_{0}
$$

Following the procedure described in a previous work of some of us ${ }^{15}$, the inplane stiffness of the material along $x$ and $y$ directions are defined as Equation (4), 
where $S_{0}$ is the unstretched area of the cell. Similarly, the Poisson's ratio of the material along $x$ and $y$ directions are defined as Equation (5), respectively.

$$
\begin{gathered}
C_{x}=\frac{1}{S_{0}}\left(2 c_{1}-\frac{c_{3}^{2}}{2 c_{2}}\right) ; C_{y}=\frac{1}{S_{0}}\left(2 c_{2}-\frac{c_{3}^{2}}{2 c_{1}}\right) \\
v_{x}=\frac{c_{3}}{2 c_{2}} ; v_{y}=\frac{c_{3}}{2 c_{1}}
\end{gathered}
$$

As mentioned above, these equations are only valid for linear elastic regimes up to the yield strength, which in the here explored grazynes corresponds to strains in the $\pm 2 \%$ range, and by that, contemplating both stretched and compressed situations. At larger strains the grazynes behave in a highly anisotropic fashion, and for these cases a nonlinear elastic analysis has been done up to the Ultimate Tensile Strength (UTS) following the continuum perspective procedure as outlined by Wei and coworkers ${ }^{25}$ and detailed in Section 3.2.

Finally, outstanding electronic properties such as an extremely high carrier mobility and charge transport arise from the presence of Dirac points in a 2D material electronic structure, as happens in graphene. Near any Dirac point the electronic energy is linear with respect to the vector displacements in the reciprocal space, so the $2 \mathrm{D}$ band structure in this region can be adjusted by Equation (6). In the case of grazynes, Dirac points have been located (see below), and in order to compare their conductivity capacity among them, and, also with respect graphene and graphynes, their Fermi velocities have been evaluated.

$$
\mathrm{E}= \pm \hbar \mathrm{v}_{\mathrm{F}} \mathbf{k}
$$

\section{RESULTS AND DISCUSSION}

3.1. Energetic and Structural Properties. Figure 4 shows the DFT PBE-D2 optimized structures for $[n],[m]$-grazynes with $n=1,2$, and 3 . The corresponding 
cohesive energies and geometric parameters are listed in Table 1 and Table 2, respectively.

Figure 4. DFT PBE-D2 optimized cells for [1],[1]-, [2],[1]-, and [3],[1]-grazynes (left, middle, and right, respectively). The $s p$ and $s p^{2}$ carbon atoms are represented by gray and black spheres, respectively. The optimized cell parameters, $a$ and $b$, are $2.553 \AA$ and $6.812 \AA$ for [1],[1]-grazyne, $2.526 \AA$ and $17.901 \AA$ for [2],[1]-grazyne, and $2.512 \AA$ and $11.084 \AA$ for [3],[1]-grazyne, respectively.
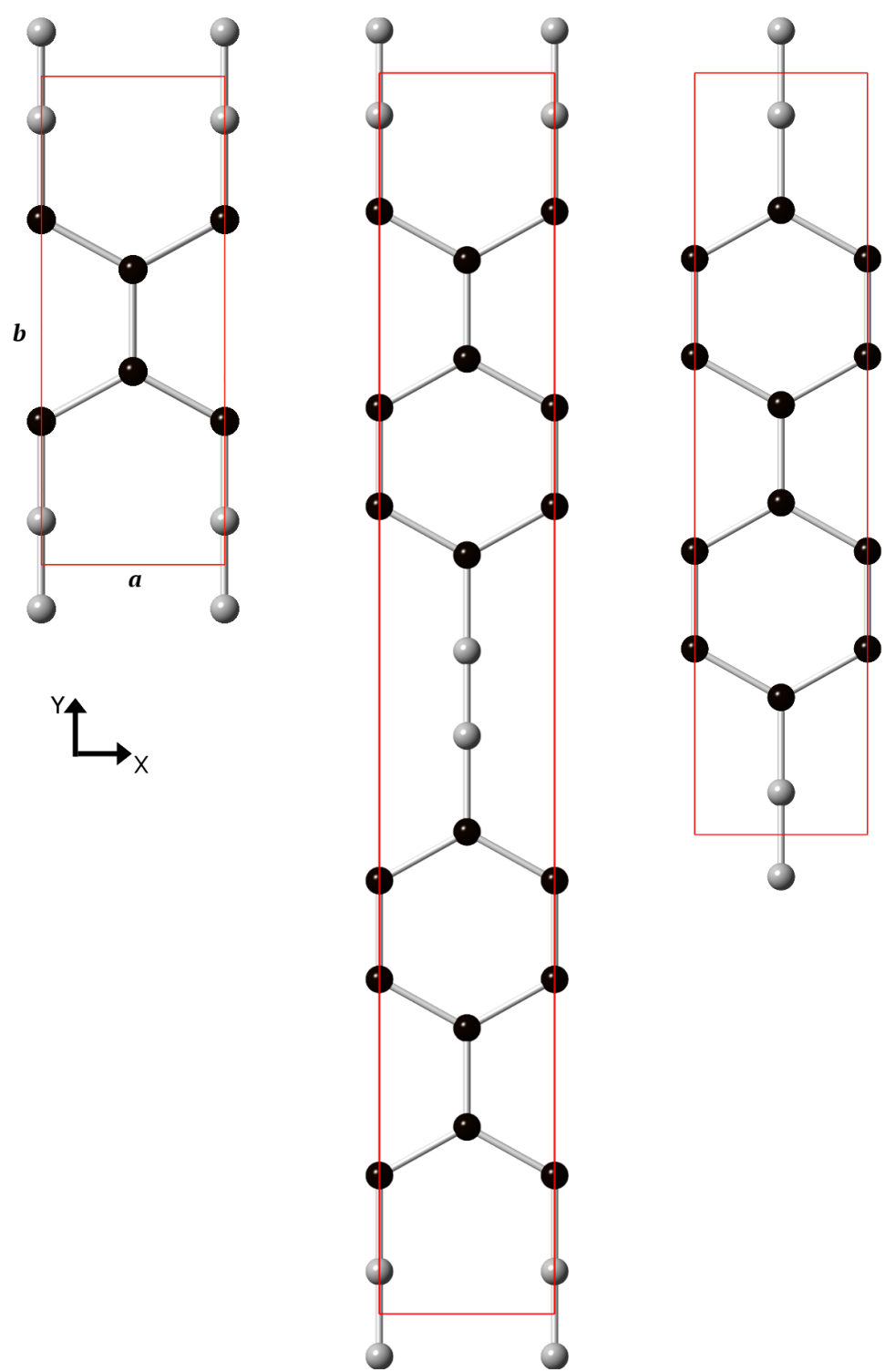
The geometric parameters obtained by self-consistent PBE optimizations using the all electron FHI-AIMS code correspond to nearly identical structures compared to VASP optimizations, with deviations in bond lengths no more than $0.01 \AA$, and energy differences below $0.02 \mathrm{eV}$ for the $E_{c o h}$. Consequently, in the following, only VASP results are discussed for this matter. Notice in Table 1 and Table 2 that cohesive energy values for $[n],[m]$-grazynes are closer to the value of graphene ${ }^{26}$ rather than to the value of graphynes, ${ }^{15}$ denoting the grazynes stability.

Table 1. DFT cohesive energy at PBE-D2 level for the optimized [ $n]$,[1]-grazynes $(n=$ 1, 2, and 3) structures and other C-based 2D materials.

$$
E_{c o h} / \mathrm{eV} \text { atom }^{-1}
$$

\begin{tabular}{cc}
\hline 1$],[1]$-grazyne & 7.36 \\
{$[2],[1]$-grazyne } & 7.49 \\
{$[3],[1]$-grazyne } & 7.58 \\
graphene $^{26}$ & 7.85 \\
$\alpha_{\text {-graphyne }}^{15}$ & 6.93 \\
$\beta$-graphyne & 7.01 \\
$\gamma$-graphyne & \\
\hline
\end{tabular}


Table 2. Bond distances in Å of DFT PBE-D2 optimized [n],[1]-grazynes ( $n=1,2$, and 3) structures. Values for graphene and $\alpha-, \beta$-, and $\gamma$-graphyne are also shown for comparison.

\begin{tabular}{|c|c|c|c|}
\hline & $\mathrm{d}(s p-s p)$ & $d\left(s p^{2}-s p\right)$ & $\begin{array}{c}\mathrm{d}\left(s p^{2}-s p^{2}\right) \\
(x-, y-) \text { direction }\end{array}$ \\
\hline [1],[1]-grazyne & 1.226 & 1.383 & $1.455,1.423$ \\
\hline [2],[1]-grazyne & 1.225 & 1.385 & $1.445,1.424$ \\
\hline [3],[1]-grazyne & 1.226 & 1.385 & $1.440,1.424$ \\
\hline graphene $^{26}$ & - & - & 1.420 \\
\hline$\alpha$-graphyne ${ }^{15}$ & 1.230 & 1.395 & - \\
\hline$\beta$-graphyne ${ }^{15}$ & 1.232 & 1.389 & 1.456 \\
\hline$\gamma$-graphyne ${ }^{15}$ & 1.222 & 1.407 & 1.426 \\
\hline
\end{tabular}

The simplest grazyne structure, the [1],[1]-grazyne is equivalent to the previously studied undistorted squarographene structure. ${ }^{27}$ The present relative stability with respect graphene per $\mathrm{C}$ atom, $\delta \mathrm{E}$, is here computed to be $0.49 \mathrm{eV}$ atom ${ }^{-1}$, which is very close to the previously reported value of $0.54 \mathrm{eV}$ atom $^{-1}$ obtained through Density Functional based Tight Binding (DF-TB) simulations. Further than that, the closer energetic relative stability found here for grazynes, being in between graphene and graphynes, is also in accordance to previous findings. ${ }^{27}$ As expected, here we found that, the higher the $n$ value, the closer to graphene stability is the $E_{c o h}$. Notice how, ultimately, graphene is only $0.27 \mathrm{eV}$ per $\mathrm{C}$ atom more stable than [3],[1]-grazyne.

It is worth to stress out that other carbon allotropes like graphynes present markedly lower cohesive energies (e.g., 6.93, 7.01, and 7.21 $\mathrm{eV}^{\text {atom }}{ }^{-1}$ for $\alpha-, \beta-$, and $\gamma-$ graphyne, respectively). These values reinforce the higher stability of [1],[1]-grazyne with respect to graphynes, and consequently, their feasibility and stability when 
synthesized and isolated, as this has been already achieved for the less stable graphyne structures. $^{28}$ Thus, their synthesis becomes only a problem of organic synthesis, although a feasible procedure could well start from iodine or bromine capped graphene stripes, ${ }^{29}$ where there is a plethora of procedures to achieve them, either through bottom-up or top-down strategies. ${ }^{30}$ Such halide-capped graphene stripes can be linked to acetylenic building blocks through Sonogashira cross-coupling reactions, ${ }^{28}$ see Figure 5.

Figure 5. Example of Sonogashira's cross-coupled reaction in between halide-capped graphene stripes and acetylene.

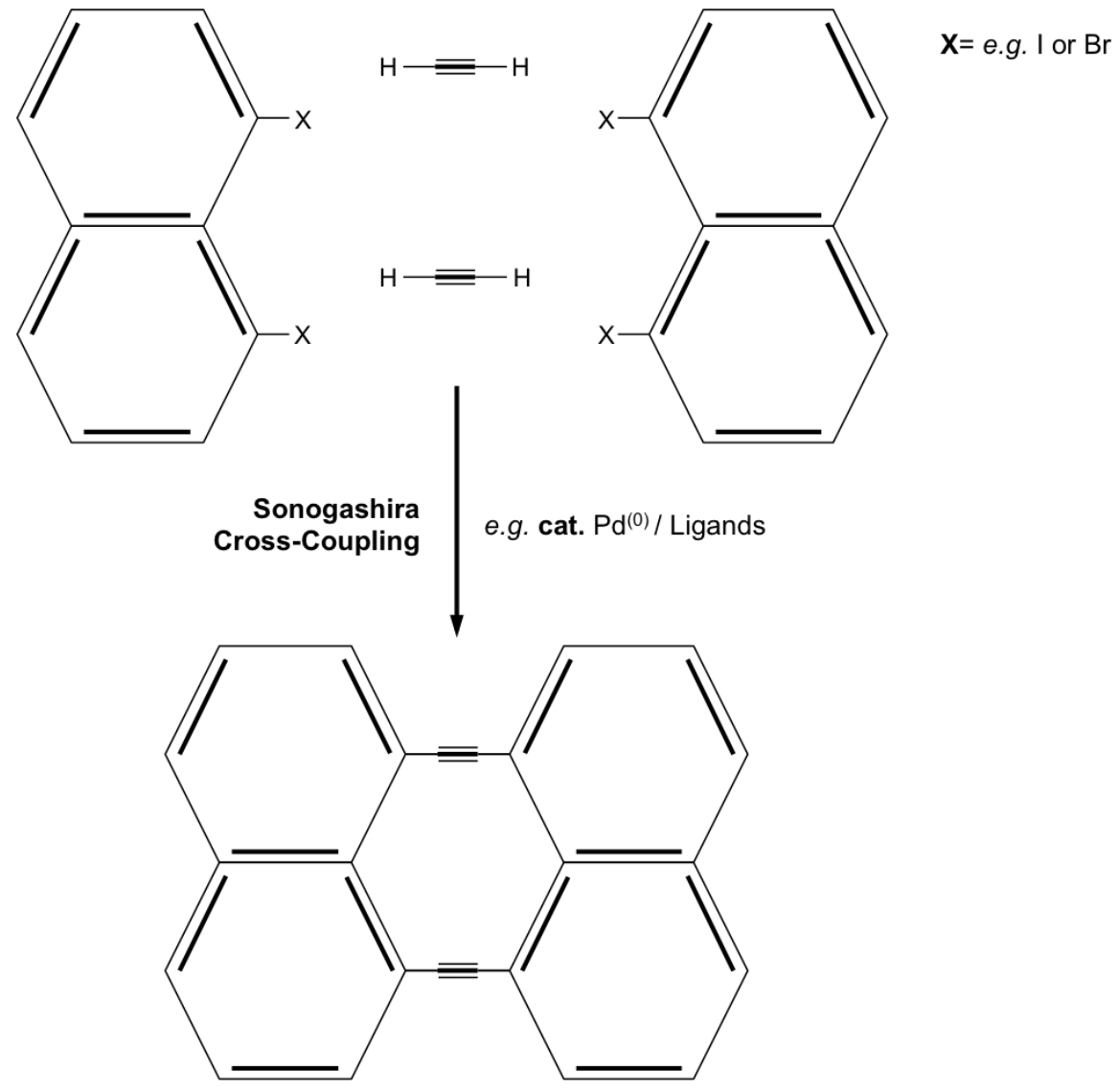


Actually, such a procedure can be step-wise controlled by protective trimethylsilylacetylene (TMS) endings applied either on the graphene stripes or the acetylenic linkages, which offer diverse interesting routes for knitting them. ${ }^{31}$ However, there are other possible synthetic routes. As an example, it is worth highlighting the similarities with the bottom-up synthesis of nanoporous graphene, as it resembles the here presented grazynes, but with $s p^{2}$ type of links in between the graphene stripes. ${ }^{32}$ Briefly, there, a precursor reactant, namely the diphenyl-10,10'-dibromo-9,9'bianthracene (DP-DBBA), was used to create graphene ribbons (stripes) through Ullmann coupling and cyclohydrogenation. The resulting graphene stripes were actually transversally linked directly through dehydrogenative cross coupling. All in all, the above successful synthesis allows stating that, although the grazynes synthesis may appear challenging, seems at hand, nevertheless.

Leaving the possible synthetic routes apart, and focusing on the grazynes geometric structure, as far as the rectangular unit cell parameters $(a, b)$ are concerned, their values are 2.553 and $6.812 \AA$ for [1],[1]-grazyne, thus very similar to the previously optimized DF-TB values of 2.51 and $6.96 \AA$, respectively. ${ }^{27}$ Such $a, b$ values are 2.526 and $17.901 \AA$ for [2],[1]-grazyne, and 2.512 and $11.084 \AA$ for [3],[1]-grazyne, respectively. These values indicate that the unit cell gets slightly compressed in $x$ direction as the $[n]$ index increases, which could well reflect the fact that the addition of a higher proportion of acetylenic linkages may disrupt the graphenic network, weakening it, and, by that, forcing its expansion.

Table 2 shows some bond distances of the optimized structures. The distances between $s p$-sp and between $s p^{2}-s p \mathrm{C}$ atoms in [1],[1]-, [2],[1]-, and [3],[1]-grazyne structures are in line with the values obtained for $\alpha$-, $\beta$-, and $\gamma$-graphyne. Indeed, the $d(s p-s p), d\left(s p^{2}-s p\right), d\left(s p^{2}-s p^{2}\right)$ distances of [1],[1]-grazyne of $1.226 \AA, 1.383 \AA$, and 
1.423-1.455 A compare well with the previously obtained DF-TB values on undistorted squarographene (i.e., $1.236 \AA$, $1.426 \AA$ and $1.446 \AA$, respectively). The only possible caveat is that DF-TB seems to imply a weaker $s p-s p^{2}$ bond, as the distance is longer than the value obtained using PBE-D2.

Back to the grazyne family analysis, the $d\left(s p^{2}-s p^{2}\right)$ distances are closer to those of graphene although two values can be distinguished, depending on the orientation of the bond. Thus, bonds in the $y$ - direction (i.e., armchair direction) are graphene like bonds and their values, only $0.3 \%$ larger than those in graphene, do not depend on the size $n$ of the stripes. Instead, the $s p^{2}-s p^{2}$ in the $x$ - direction (i.e., zig-zag direction) bonds are larger than graphene like bonds and their values are reduced as the size of the stripe increases. Thus, zig-zag $s p^{2}-s p^{2}$ bonds are $2.5 \%, 1.8 \%$, and $1.4 \%$ larger for [1],[1]-, [2],[1]-, and [3],[1]-grazynes than in graphene, respectively.

3.2. Elastic Properties. For the above commented structures, we analyzed in detail the elastic constants in the linear regime according to Equation (3), thus applying $\varepsilon_{x}$ (in $x$ - direction) and $\varepsilon_{y}$ (in $y$ - direction) strains in the range $\pm 2 \%$ to the cells shown in Figure 4. To do so the lattice constants were changed with $0.5 \%$ steps along both directions (i.e., $\varepsilon_{x}=\Delta a / a_{0}$ and $\varepsilon_{y}=\Delta b / b_{0}$, with $a_{0}$ and $b_{0}$ being the cell parameters corresponding to the minimum energy structure, Figure 4). Thus, a total of 81 DFT points were used to fit the previous $E=E\left(\varepsilon_{x}, \varepsilon_{y}\right)$ expression (see Table 3). In isotropic 2D materials, the elastic constants along $x$ and $y$ directions are identical due to the symmetry of the lattice. Our calculations show anisotropy in the Poisson's ratio and the in-plane stiffness along $x$ - (parallel direction) and $y$-(perpendicular direction). This is expected given the presence of acetylenic linkages that break the structural symmetry. The obtained values of $c_{1}, c_{2}$, and $c_{3}$, along with the minimum $E_{0}$ and the exposed surface, $S_{0}$, are shown in Table 3. 
Table 3. Fitted parameters for PBE-D2 energy as a function of the strain applied in $x$ and $y$-directions $\left(E=c_{1} \varepsilon_{x}^{2}+c_{2} \varepsilon_{y}^{2}+c_{3} \varepsilon_{x} \varepsilon_{y}+E_{0}\right)$, as applied on [n],[1]-grazynes $(n=$ 1,2 , and 3) structures, along with the exposed surface area, $S_{0}$, and the adjustment Root Mean Squared Deviation (RMSD).

\begin{tabular}{cccc}
\hline Fitted parameters & {$[\mathbf{1}],[\mathbf{1}]$-grazyne } & [2],[1]-grazyne & [3],[1]-grazyne \\
\hline$c_{1} / \mathrm{eV}$ & 122.12294 & 358.55816 & 234.17391 \\
$c_{2} / \mathrm{eV}$ & 207.70522 & 528.08010 & 321.65298 \\
$c_{3} / \mathrm{eV}$ & 47.39527 & 136.17870 & 91.93225 \\
$E_{0} / \mathrm{eV}$ & -52.39949 & -141.85390 & -89.47430 \\
$S_{0} / \AA^{2}$ & 17.39104 & 45.21793 & 27.84301 \\
$\mathrm{RMSD}$ & $9.22 \cdot 10^{-3}$ & $2.05 \cdot 10^{-2}$ & $1.44 \cdot 10^{-2}$ \\
\hline
\end{tabular}

With the adjusted parameters, shown in Table 3, the Poisson's ratio and the inplane stiffness can be obtained, according to Equations (4) and (5), respectively, in a straightforward fashion, and these have been gained and gathered in Table 4. From their inspection it is interesting to note that [1],[1]- and [2],[1]-grazyne materials are stiffer than graphene in $y$-direction, essentially due to the presence of acetylenic units in this direction $^{33,34,35}$. As the size of the graphenic stripes increases, the in-plane stiffness in both directions becomes closer. One would expect that these two values would converge towards the graphene value for higher values of $n$. In the case of Poisson's ratios, the highest value is also obtained in $x$ - direction, with values around 0.19 close to the experimental value of graphene ${ }^{25}$. The in-plane stiffness for $[n],[m]$-grazynes in both directions are also above the values for graphynes ${ }^{36,37}($ e.g., $21.98,73.07$, and $165.51 \mathrm{~N}$ $\mathrm{m}^{-1}$ for $\alpha-, \beta$-, and $\gamma$-graphyne, ${ }^{15}$ respectively) whereas the Poisson's ratio values of grazynes are below those of graphynes ${ }^{38}$ (e.g., $0.87,0.67$, and 0.42 for $\alpha-, \beta-$, and $\gamma$ - 
graphyne, ${ }^{15}$ respectively). Our in-plane stiffness values for [1],[1]-grazyne in $x$ - and $y$ direction, $\mathrm{C}_{\mathrm{x}}=220.03 \mathrm{~N} \mathrm{~m}^{-1}$ and $\mathrm{C}_{\mathrm{y}}=374.23 \mathrm{~N} \mathrm{~m}^{-1}$, are in agreement with those reported by Sun et al. of $\mathrm{C}_{\mathrm{x}}=203.5 \mathrm{~N} \mathrm{~m}^{-1}$ and $\mathrm{C}_{\mathrm{y}}=351.7 \mathrm{~N} \mathrm{~m}^{-1}$, respectively. ${ }^{39}$

Table 4. Elastic properties (Poisson's ratio and in-plane stiffness in $x$ - and $y$-direction) for $[n],[1]$-grazynes $(n=1,2$, and 3). Values for graphene and $\alpha-, \beta$-, $\gamma$-graphyne are also shown for comparison.

\begin{tabular}{|c|c|c|c|c|}
\hline & \multicolumn{2}{|c|}{ Poisson's ratio } & \multicolumn{2}{|c|}{ In-plane stiffness / $\mathrm{N} \mathrm{m}^{-1}$} \\
\hline & $v_{x}$ & $v_{\mathbf{y}}$ & $\mathbf{C}_{\mathbf{x}}$ & $\mathbf{C}_{\mathbf{y}}$ \\
\hline [1],[1]-grazyne & 0.194 & 0.114 & 220.03 & 374.23 \\
\hline [2],[1]-grazyne & 0.190 & 0.129 & 247.87 & 365.06 \\
\hline [3],[1]-grazyne & 0.196 & 0.143 & 261.94 & 359.80 \\
\hline \multirow[t]{2}{*}{ graphene } & \multicolumn{2}{|c|}{$0.18^{15}, 0.149^{33}$} & \multicolumn{2}{|c|}{$341.09^{15}, 363^{33}, 347^{36}$} \\
\hline & \multicolumn{2}{|c|}{$0.169^{25}$} & \multicolumn{2}{|c|}{$340^{34}, 348^{25}$} \\
\hline \multirow[t]{2}{*}{$\alpha$-graphyne } & \multicolumn{2}{|c|}{$0.87^{15}, 0.88^{26}$} & \multicolumn{2}{|c|}{$21.98^{15}, 21^{26}$} \\
\hline & \multicolumn{2}{|c|}{$0.874^{33}$} & & \\
\hline$\beta$-graphyne & \multicolumn{2}{|c|}{$0.67^{15}$} & \multicolumn{2}{|c|}{$73.07^{15}$} \\
\hline \multirow[t]{2}{*}{$\gamma$-graphyne } & \multicolumn{2}{|c|}{$0.42^{15}, 0.479^{33}$} & \multicolumn{2}{|c|}{$165.51^{15}, 178^{33}, 166^{36}$} \\
\hline & $0.417^{36}, 0.429^{37}$ & $\begin{array}{l}429^{37} \\
35\end{array}$ & \multicolumn{2}{|c|}{$170-224^{38}, 162.1^{26}, 166.3^{35}$} \\
\hline
\end{tabular}

At higher strains grazynes show a clear nonlinear elastic behavior, though. Consequently, their nonlinear elastic constants were evaluated using the fourth order continuum description of the nonlinear elasticity theory, ${ }^{25}$ where the material elastic properties are determined from the elastic strain energy density, $\Phi$, quadratic in strain for a linear elastic material. To account for the nonlinear elastic behavior $\Phi$ is expanded in a Taylor series in terms of powers of strain, as 


$$
\Phi=\frac{1}{2 !} C_{i j k l} \eta_{i j} \eta_{k l}+\frac{1}{3 !} C_{i j k l m n} \eta_{i j} \eta_{k l} \eta_{m n}+\frac{1}{4 !} C_{i j k l m n o p} \eta_{i j} \eta_{k l} \eta_{m n} \eta_{o p}+\cdots
$$

where lower case subscripts range from 1 to $3, \eta_{i j}$ is the Lagrangian elastic strain, related to the true strain by $\eta=\frac{1}{2}\left[(\varepsilon+1)^{2}-1\right]$ and $\mathrm{C}$ represents each higher-order elastic modulus tensor whose rank corresponds to the number of subscripts (i.e., the Second-Order Elastic Constants (SOEC), $C_{i j k l}$, and the Third- and the Fourth-Order Elastic Constants (TOEC and FOEC, respectively) are given by the components of the fourth-, sixth-, and eight-rank tensors, respectively).

The symmetric second Piola-Kirchoff $(\mathrm{P}-\mathrm{K})$ stress tensor, $\Sigma_{i j}$, is derived as

$$
\Sigma_{i j}=\frac{\partial \Phi}{\partial \eta_{i j}}=C_{i j k l} \eta_{k l}+\frac{1}{2 !} C_{i j k l m n} \eta_{k l} \eta_{m n}+\frac{1}{3 !} C_{i j k l m n o p} \eta_{k l} \eta_{m n} \eta_{o p} \cdots
$$

and employing the Voigt $^{40}$ notation (i.e., $11 \rightarrow 1,22 \rightarrow 2,33 \rightarrow 3,23 \rightarrow 4,31 \rightarrow 5$ and $12 \rightarrow 6$ ), both equations can be rewritten as

$$
\begin{gathered}
\Phi=\frac{1}{2 !} C_{I J} \eta_{I} \eta_{J}+\frac{1}{3 !} C_{I J K} \eta_{I} \eta_{J} \eta_{K}+\frac{1}{4 !} C_{I J K L} \eta_{I} \eta_{J} \eta_{K} \eta_{L}+\cdots \\
\Sigma_{I}=\frac{\partial \Phi}{\partial \eta_{I}}=C_{I J} \eta_{J}+\frac{1}{2 !} C_{I J K} \eta_{J} \eta_{K}+\frac{1}{3 !} C_{I J K L} \eta_{J} \eta_{K} \eta_{L}+\cdots
\end{gathered}
$$

with an upper-case summation subscript running from 1 to 6 . Since the deformed state of grazynes has no bending contribution only in-plane stress and strain components can be considered (i.e., components with subscripts 1 and/or 2).

Hence, the mechanical response under uniaxial Lagrangian strain ( $\eta)$ along the $x$ direction (i.e., $\eta_{x}=\eta_{1} \geq 0, \eta_{y}=\eta_{2}=\eta_{6}=0$ ) can be represented by

$$
\begin{aligned}
& \sum_{1}^{1}=c_{11} \eta_{1}+\frac{1}{2} c_{111} \eta_{1}^{2}+\frac{1}{6} c_{1111} \eta_{1}^{3} \\
& \sum_{2}^{1}=c_{12} \eta_{1}+\frac{1}{2} c_{112} \eta_{1}^{2}+\frac{1}{6} c_{1112} \eta_{1}^{3}
\end{aligned}
$$


whereas, the uniaxial Lagrangian strain $(\eta)$ along the $y$ direction (i.e., $\eta_{x}=\eta_{1}=\eta_{6}=0$, $\left.\eta_{y}=\eta_{2} \geq 0\right)$ follows,

$$
\begin{aligned}
& \sum_{1}^{2}=c_{12} \eta_{2}+\frac{1}{2} c_{122} \eta_{2}^{2}+\frac{1}{6} c_{1222} \eta_{2}^{3} \\
& \sum_{2}^{2}=c_{22} \eta_{2}+\frac{1}{2} c_{222} \eta_{2}^{2}+\frac{1}{6} c_{2222} \eta_{2}^{3}
\end{aligned}
$$

Finally, the equi-biaxial loading can be represented by

$$
\begin{aligned}
& \sum_{1}^{\text {biax }}=\left(c_{11}+c_{12}\right) \eta+\frac{1}{2}\left(c_{111}+c_{122}+2 c_{112}\right) \eta^{2}+\frac{1}{6}\left(c_{1111}+c_{1222}+3 c_{1112}+\right. \\
& \left.3 c_{1122}\right) \eta^{3} \\
& \sum_{2}^{\text {biax }}=\left(c_{22}+c_{12}\right) \eta+\frac{1}{2}\left(c_{112}+c_{222}+2 c_{122}\right) \eta^{2}+\frac{1}{6}\left(c_{1112}+c_{2222}+3 c_{1222}+\right. \\
& \left.3 c_{1122}\right) \eta^{3}
\end{aligned}
$$

From VASP calculations, the true Cauchy stress $(\boldsymbol{\sigma})$ is obtained at different strains. Then, the stress is converted to the second P-K stress $\Sigma$ through the deformation tensor $(\boldsymbol{F})$ by means of,

$$
\boldsymbol{\Sigma}=J \boldsymbol{F}^{-1} \boldsymbol{\sigma}\left(\boldsymbol{F}^{-1}\right)^{T}
$$

where $J$ is the deformation tensor determinant.

The nonlinear elastic constants were evaluated by performing a least-squares fitting of the stress-strain data to Equations (11)-(15). Different magnitudes of uni- and biaxial strains in the $x$ - and $y$-directions were applied on the grazyne cells (see Figure 1 for cell orientation), dilating the unit cells along the loading direction and applying an equal affine transformation to the atomic positions. The deformed cell was posteriorly minimized keeping constant the cell dimensions but allowing the atoms relaxing in the plane. 
Figures 6 and 7 show the true stress-strain curves and the second P-K stresses, respectively, for the grazyne structures at uniaxial $x$ - and $y$-strains, along with the equibiaxial strain. Figure 7 also shows the best fits obtained for Equations (11)-(15) at strains up to the UTS. Under uniaxial strain in $x$-direction ( $y$-direction) the UTS corresponds to $18.93(29.08), 21.71(28.50)$, and $23.44(28.61) \mathrm{N} \mathrm{m}^{-1}$ for [1],[1]-, [2],[1]- and [3],[1]-grazynes, respectively, at $\eta_{x}\left(\eta_{y}\right)=0.22(0.17)$. Thus, grazynes withstand higher stresses in the $y$-direction due to the presence of acetylenic linkages. Moreover, increasing the stripes width enables the material resist higher stresses in both directions. Table 5 lists the elastic coefficients for the analyzed grazyne structures. Obviously, the second order elastic constants obtained here from stress-strain data are equivalent to those listed in Table 3 that were obtained in the harmonic analysis of strain energy although considering that $c_{1}=\frac{1}{2} c_{11}, c_{2}=\frac{1}{2} c_{22}$, and $c_{3}=c_{12}$. 
Figure 6. True Cauchy stress vs. strains, $\sigma_{x}$ and $\sigma_{y}$, for [1],[1]- (red circles), [2],[1](blue squares), and [3],[1]-(green rhombus) grazyne structures for uniaxial tension in $x$ direction, $\varepsilon_{x}$ (top), uniaxial tension in $y$-direction, $\varepsilon_{y}$ (middle), and equi-biaxial tension, $\varepsilon$ (bottom).
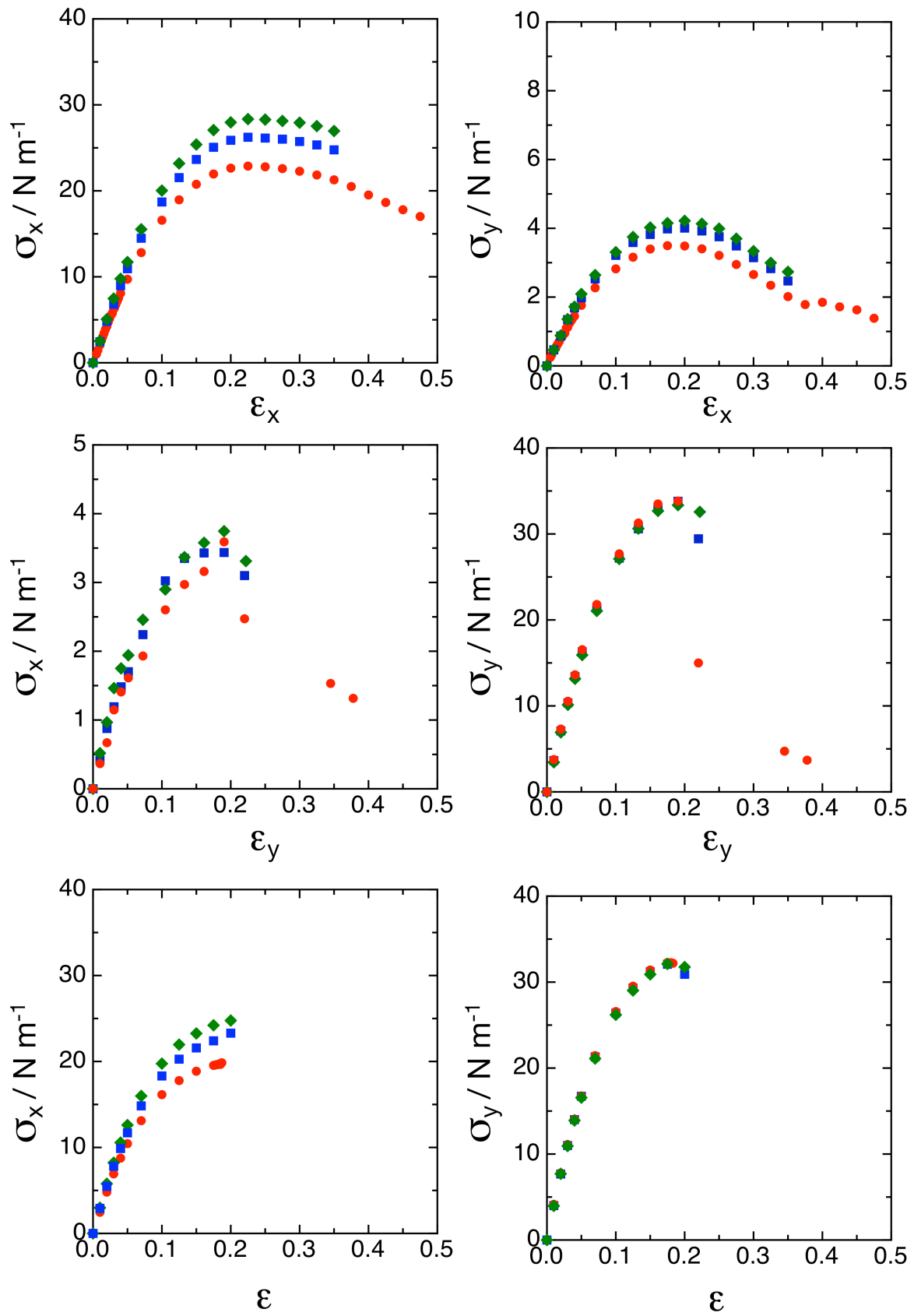
Figure 7. Second Piola-Kirchoff stress vs. lagrangian strain for [1],[1]- (circles), [2],[1](squares), and [3],[1]- (rhombus) grazyne structures for uniaxial tension in $x$-direction, $\eta_{1}=\eta_{x}$ (top), uniaxial tension in y-direction, $\eta_{2}=\eta_{y}$ (middle) and equi-biaxial tension, $\eta$ (bottom). Solid lines correspond to the best fits obtained for Equations (11)$(15)$.
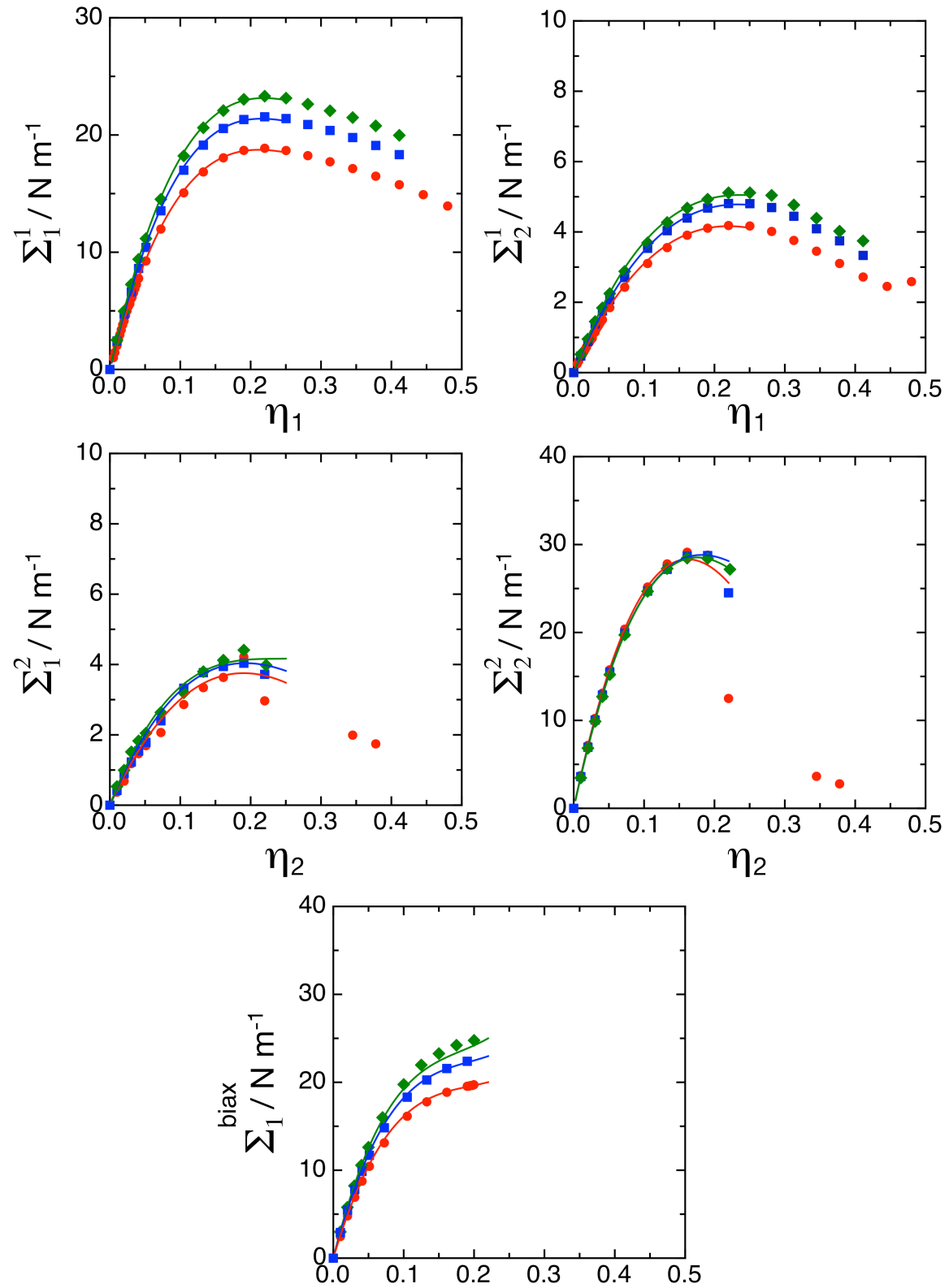

$\eta$ 
Table 5. Elastic coefficients for $[n]$,[1]-grazynes $(n=1,2$, and 3$)$ structures obtained from fitting second Piola-Kirchoff stresses shown in Figure 6 with Equations (11)-(15).

\begin{tabular}{|c|c|c|c|c|}
\hline Elastic constants & $/ \mathbf{N ~ m}^{-1}$ & {$[1],[1]$-grazyne } & {$[2],[1]$-grazyne } & {$[3],[1]$-grazyne } \\
\hline \multirow{3}{*}{$\begin{array}{l}\text { U } \\
\text { 멍 } \\
\text { W }\end{array}$} & $c_{11}$ & 225.02 & 254.09 & 269.50 \\
\hline & $c_{22}$ & 382.70 & 374.22 & 370.18 \\
\hline & $c_{12}$ & 43.66 & 48.25 & 52.90 \\
\hline \multirow{4}{*}{$\begin{array}{l}\underset{\text { II }}{O} \\
\stackrel{\bullet}{*}\end{array}$} & $c_{111}$ & -1742.68 & -1952.87 & -2025.93 \\
\hline & $c_{222}$ & -2977.22 & -3006.33 & -2878.45 \\
\hline & $c_{112}$ & -277.46 & -300.02 & -353.29 \\
\hline & $c_{122}$ & -295.33 & -348.88 & -449.58 \\
\hline \multirow{5}{*}{ 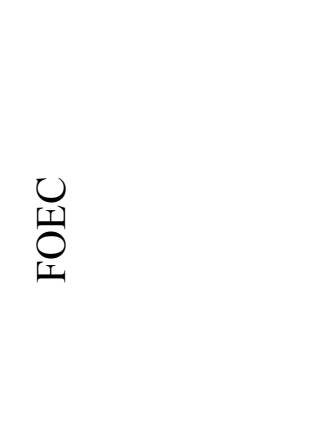 } & $c_{1111}$ & 6419.82 & 7179.95 & 7259.93 \\
\hline & $c_{2222}$ & 7609.00 & 10451.24 & 8762.75 \\
\hline & $c_{1112}$ & 717.02 & 798.60 & 1097.56 \\
\hline & $c_{1222}$ & 689.77 & 1021.10 & 1916.38 \\
\hline & $c_{1122}$ & 977.64 & 240.51 & 634.52 \\
\hline
\end{tabular}

3.3. Electronic Properties. Regarding the electronic properties of the studied grazynes, these have been obtained following the k-space paths defined in Figure 3 . The obtained band structures and the corresponding Density of States (DOS) for [1],[1]-, [2],[1]-, and [3],[1]-grazynes are shown in Figure 8. 
Figure 8. Electronic band structures of [1],[1]-, [2],[1]-, and [3],[1]-grazyne structures (top, middle, and bottom, respectively) along with the DOS diagram. Red line delimits the Fermi energy, $\mathrm{E}_{\mathrm{F}}$, arbitrarily set to zero.

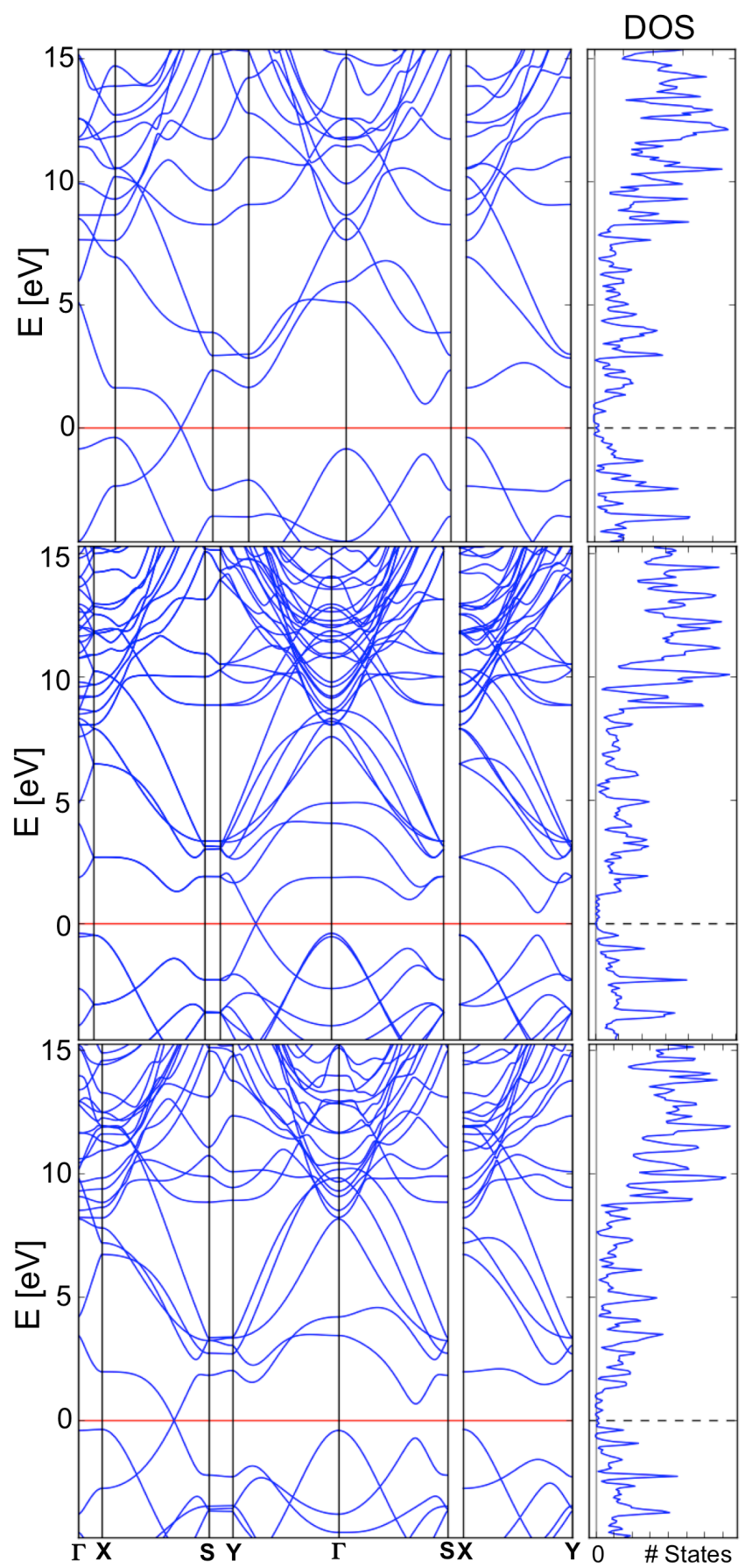


It is worth to stress out that, at variance with PBE-D2 and other DFT based methods, the employment of PBE0 hybrid functional avoids the known underestimation of energy band gaps. ${ }^{9}$ This fact reinforces the point that the shown Dirac points (or Dirac cones) located at Fermi energies, $\mathrm{E}_{\mathrm{F}}$, of all the studied grazynes are no artifact of the employed methodology. Further than that, despite the band structure sampling is done only on particular directions of the reciprocal space, and so, not sampling the full k-space, it seems as the found Dirac cones are so, given that the full integration of the reciprocal space, as carried out on the projected DOS, reveals a nominally zero density of states at Fermi energy. This already implies that the last valence and first conduction bands do not cross the Fermi level in other points of the reciprocal space out of the explored regions. The present results differ from the previous DF-TB results on undistorted squarographene, ${ }^{27}$ as there, only an open Dirac cone in the $\mathbf{X} \rightarrow \mathbf{S}$ path was found, with a direct band gap of $0.7 \mathrm{eV}$. However, in such previous results, a nominally zero bandgap was extracted from the DOS. It seems, as the DF-TB was not capable of properly describing the peculiarities of such band structures, which unduly lead to the description of undistorted squarographene as a semiconductor. The here presented PBE0 results show that, regardless of the studied grazyne, Dirac cones are present in $\mathbf{X} \rightarrow \mathbf{S}$ ([1],[1]- and [3],[1]-grazynes) or $\mathbf{Y} \rightarrow \Gamma$ [2],[1]-grazyne) paths.

The systems feature linear band dispersion in the proximity of the Dirac cones, as characteristic of graphene and graphynes. As commented, in two cases they are located across the $\mathbf{X} \rightarrow \mathbf{S}$ or $\mathbf{Y} \rightarrow \Gamma$ paths, which are placed along the graphene stripe direction. Indeed, no Dirac cones are featured along the graphyne direction, with just a succinct approach of bands near the $\mathbf{X}$ point. Therefore, it seems, as the ballistic transport is achievable along the graphene stripes channels, but inhibited perpendicular to it, pointing for anisotropy in the charge carrier mobility, with fundamental implications in oriented nanoelectronic devices. 
One may wonder whether strain affects the grazynes band structures. To this end, the electronic structure has been evaluated for [1],[1]-grazyne when compressed/stretched in the elastic region by $\pm 2 \%$ along the graphenic $(x)$ and acetylenic $(y)$ directions, also considering the uniaxial and biaxial deformations. Figure 9 shows the results of the original band structure with no deformations, plus the eight possibilities of compressions/stretching.

From the inspection of Figure 9 several conclusions can be withdrawn: First, the overall electronic structures are marginally affected by the strain, with only some changes in the bands energetic dispersion. More importantly is that Dirac cones prevail with strain, regardless of the strain direction, the simultaneous strains along different axes, even opposite strain directions on different axes. This implies that such Dirac cones are resilient to such in-plane deformations. If any, only some minor changes are detected. The above-commented band at $\mathbf{X}$ approaches the $\mathrm{E}_{\mathrm{F}}$ when compressed along the graphenic direction, and separates when stretched, with marginal effect when the strain is applied in the acetylenic direction.

Given the above, the Fermi velocities in the proximity of the Dirac cones have been calculated using Equation (6) on the unstrained geometry, and employing five points located in the range of $\pm 0.04 \AA^{-1}$ in the fitting procedure. The Fermi velocity values, listed in Table 6, are slightly smaller than the Fermi velocities, calculated likewise, for graphene and graphynes. However, as the width of the graphene stripes increases $(e . g ., n=1,2,3, \ldots)$, the Fermi velocities become closer to that of graphene. In any case, grazynes can be still regarded as materials with directional ballistic transport. Another aspect of interest is that graphenic stripes are actually isolated, as happens on H-capped graphene nanoribbons reported in the literature, which present edge effects that perturb their electronic structure, even more when non-capped. ${ }^{41}$ In 
this sense, the grazyne formation would be a way of displaying a semimetal character on very thin graphene stripes, without losing too much conductivity in the process.

Figure 9. Electronic band structures of [1],[1]-grazyne structures while compressed/ stretched $\pm 2 \%$ along graphenic $(x)$ and acetylenic $(y)$ directions. Red line delimits the Fermi energy, $\mathrm{E}_{\mathrm{F}}$, arbitrarily set to zero.

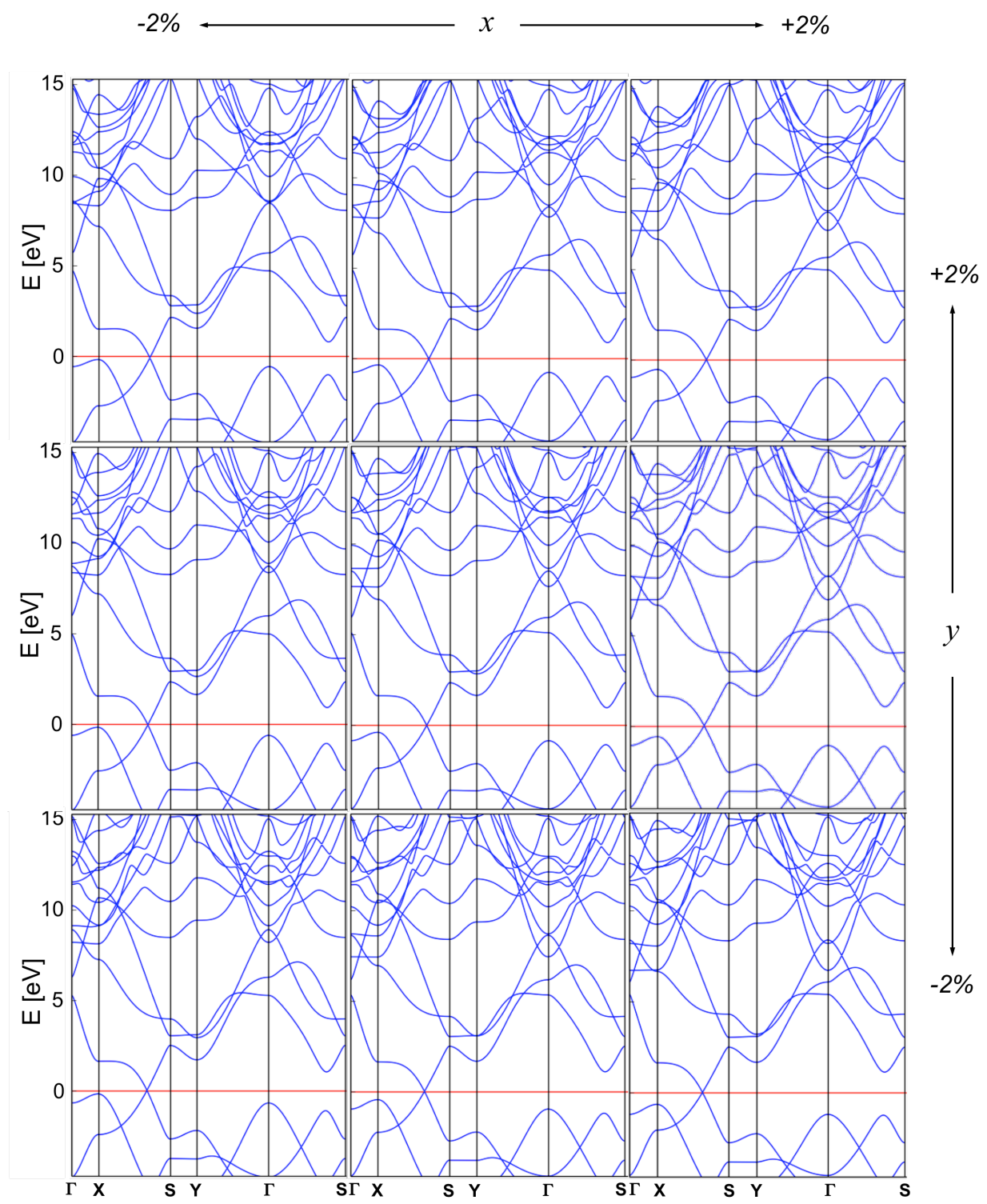


Table 6. Calculated PBE0 Fermi velocities, in $\mathrm{m} \cdot \mathrm{s}^{-1}$, for $[n]$, [1]-grazynes $(n=1,2$, and 3) Dirac cones, and their location in k-space (in parentheses, see Figure 3). Values for graphene and $\alpha$-, and $\beta$-graphyne are shown for comparison, yet previously obtained at DFT PBE level.

\begin{tabular}{cc} 
& Fermi velocity \\
\hline 1$],[1]$-grazyne & $4.81 \cdot 10^{5}(\mathrm{X} \rightarrow \mathrm{S})$ \\
{$[2],[1]$-grazyne } & $4.85 \cdot 10^{5}(\mathrm{Y} \rightarrow \Gamma)$ \\
{$[3],[1]$-grazyne } & $5.02 \cdot 10^{5}(\mathrm{X} \rightarrow \mathrm{S})$ \\
graphene $^{26}$ & $8.3 \cdot 10^{5}(\Gamma)$ \\
$\alpha$-graphyne $^{15}$ & $6.76 \cdot 10^{5}(\mathrm{~K})$ \\
$\beta$-graphyne & \\
\hline
\end{tabular}

\section{CONCLUSIONS}

Altogether, here we present a new family of 2D carbon allotropes based on graphene stripes knitted to each other by acetylenic linkages. Given the large amount of allowed connectivities we name them as grazynes. The results on the structural, elastic, and electronic properties of a set of simple grazynes by means of DFT calculations, including consistent calculations within GGA but also considering hybrid functionals, allow concluding that such grazynes are exotic yet stable materials, which could be experimentally achieved, being a priori more stable than already synthesized graphynes. In general terms, they are stiffer than graphene in the acetylenic direction, and present Dirac points in the reciprocal space along the graphene stripes, resilient to strain, and featuring Fermi velocities comparable to the latter. 


\section{ACKNOWLEDGMENTS}

The work was supported by Spanish Ministerio de Economía y Competitividad grants (CTQ2014-53987-R, CTQ2015-64618-R and RTI2018-094757-B-I00, MCIU/AEI/FEDER, UE) and, in part, by Generalitat de Catalunya grants (2014SGR1582, 2017SGR13, and XRQTC). P.G. thanks Generalitat de Catalunya for his Serra Húnter Associate Professorship and F.V. thanks the Spanish Ministerio de Economía y Competitividad for the Ramón y Cajal (RyC) postdoctoral grant (RYC2012-10129). Financial support from Spanish MINECO through the Excellence María de Maeztu program (grant MDM-2017-0767) is also fully acknowledged. P.G. wants to dedicate this work to his beloved sister (Graciela); all the doors hide something; grazynes were behind one. I am sure that behind another door you will find love. 
TOC Grafic

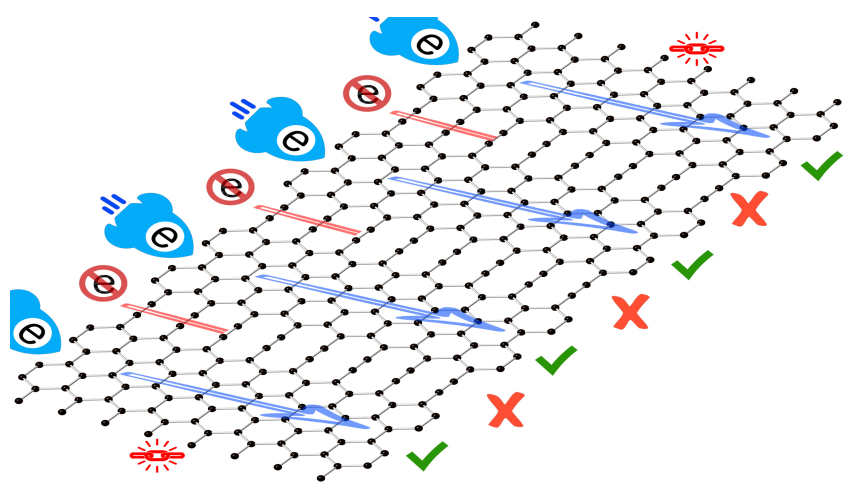




\section{REFERENCES}

(1) Osborne, I. S. The Rise and Rise of 2D Materials. Science 2006, 353, 458-460.

(2) Novoselov, K. S.; Geim, A. K.; Morozov, S. V.; Jiang, D.; Zhang, Y.; Dubonos, S. V.; Grigorieva, I. V.; Firsov, A. A. Electric Field Effect in Atomically Thin Carbon Films. Science 2004, 306, 666-669.

(3) Malko, D.; Neiss, C.; Viñes, F.; Görling, A. Competition for Graphene: Graphynes with Direction-Dependent Dirac Cones. Phys. Rev. Lett. 2012, 108, 086804.

(4) Sofo, J. O.; Chaudhari, A. S.; Barber, G. D. Graphane: a Two-dimensional Hydrocarbon. Phys. Rev. B 2007, 75, 153401.

(5) Peng, Q.; Dearden, A. K.; Crean, J.; Han, L.; Liu, S.; Wen, X.; De, S. New Materials Graphyne, Graphdiyne, Graphone, and Graphane: Review of Properties, Synthesis, and Application in Nanotechnology. Nanotechnol. Sci. Appl. 2012, 10, $1-29$.

(6) Hirsch, A.; Hauke, F. Post-graphene 2D Chemistry: the Emerging Field of Molybdenum Disulfide and Black Phosphorus Functionalization. Angew. Chem. Int. Ed. 2018, 57, 4338-4354.

(7) Li, Z.; Smeu, M.; Rives, A.; Maraval, V.; Chauvin, R.; Ratner, M. A.; Borguet, E. Towards Graphyne Molecular Electronics. Nat. Commun. 2015, 6, 6321.

(8) Alcón, I.; Bromley, S. T. Triarylmethyl-based 2D Covalent Networks: Virtual Screening of Chemical Functionalisation for Optimising Strain-induced Property Control. Phys. Chem. Chem. Phys. 2018, 20, 5028-5035.

(9) Alcón, I.; Viñes, F.; Moreira, I. dP R.; Bromley, S. T. Existence of Multi-radical and Closed-shell Semiconducting States in Post-graphene Organic Dirac Materials. Nat. Commun. 2017, 8, 1957.

(10) Steiner, C.; Gebhardt, J.; Ammon, M.; Yang, Z.; Heidenreich, A.; Hammer, N.; Görling, A.; Kivala, M.; Maier, S. Hierarchical on-Surface Synthesis and Electronic Structure of Carbonyl-Functionalized One- and Two-Dimensional Covalent Nanoarchitectures. Nat. Commun. 2017, 8, 14765.

(11)Kresse, G.; Furthmüller, J. Efficiency of ab-Initio Total Energy Calculations for Metals and Semiconductors Using a Plane-Wave Basis Set. Comput. Mater. Sci. 1996, 6, 15-50.

(12)Blum, V.; Gehrke, R.; Hanke, F.; Havu, P.; Havu, V. Ab Initio Molecular Simulations with Numeric Atom-Centered Orbitals. Comput. Phys. Commun. 2009, 180, 2175-2196.

(13)Blöchl, P. E. Projector Augmented-Wave Method. Phys. Rev. B Condens. Matter. Mater. Phys. 1994, 50, 17953.

(14)Harl, J.; Schimka, L.; Kresse, G. Assessing the Quality of the Random Phase Approximation for Lattice Constants and Atomization Energies of Solids. Phys. Rev. B 2010, 81, 115126.

(15)Puigdollers, A. R.; Alonso, G.; Gamallo, P. First-Principles Study of Structural, Elastic and Electronic Properties of $\alpha-, \beta$ - and $\gamma$-Graphyne. Carbon 2016, 96, 879887.

(16)Muñoz-Galán, H.; Viñes, F.; Gebhardt, J.; Görling, A.; Illas, F. The Contact of Graphene with Ni(111) Surface: Description by Modern Dispersive Forces Approaches. Theor. Chem. Acc. 2016, 135, 165.

(17)Wu, S.; Li, M.; Phan, H.; Wang, D.; Herng, T. S.; Ding, J.; Wu, J. Toward $\pi-$ Conjugated 2D Covalent Organic Radical Frameworks. Angew. Chem. Int. Ed. 2018, 130, 8139-8143. 
(18)Kim, S.; Gamallo, P.; Viñes, F.; Lee, J. Y. The Nano Gold Rush: Graphynes as Atomic Sieves for Coinage and Pt-group Transition Metals. Appl. Surf. Sci. 2020, 499, 143927.

(19) Monkhorst, H. J.; Pack, J. D. Special Points for Brillouin-Zone Integrations. Phys. Rev. B 1976, 13, 5188-5192.

(20)Perdew, J. P.; Burke, K.; Ernzerhof, M. Generalized Gradient Approximation Made Simple. Phys. Rev. Lett. 1996, 77, 3865-3868.

(21) Adamo, C.; Barone, V. Toward Reliable Density Functional Methods without Adjustable Parameters: the PBE0 Model. J. Chem. Phys. 1999, 110, 6158-6170.

(22)van Lenthe, E.; Baerends, E. J.; Snijders, J. G. Relativistic Total Energy Using Regular Approximations. J. Chem. Phys. 1994, 101, 9783-9792.

(23)Dunning, T. H. Jr. Gaussian Basis Sets for Use in Correlated Molecular Calculations. I. The Atoms Boron Through Neon and Hydrogen. J. Chem. Phys. 1989, 90, 1007-1023.

(24)Viñes, F.; Illas, F. Electronic Structure of Stoichiometric and Reduced ZnO from Periodic Relativistic all Electron Hybrid Density Functional Calculations Using Numeric Atom-Centered Orbitals. J. Comput. Chem. 2017, 38, 523-529.

(25)Wei, X.; Fragneaud, B.; Marianetti, C. A.; Kysar, J. W. Nonlinear Elastic Behavior of Graphene: Ab Initio Calculations to Continuum Description. Phys. Rev. B 2009, 80, 205407.

(26)Özçelik, V. O.; Ciraci, S. Size Dependence in the Stabilities and Electronic Properties of $\alpha$-Graphyne and Its Boron Nitride Analogue. J. Phys. Chem. C 2013, $117,2175-2182$.

(27)Enyashin, A. N.; Ivanovskii, A. L. Graphene Allotropes. Phys. Status Solidi B 2011, 248, 1879-1883.

(28)Zhou, J. Y.; Gao, X.; Liu, R.; Xie, X. Q.; Yang, J.; Zhang, S.; Zhang, G.; Liu, H.; Li, Y.; Zhang, J. et al. Synthesis of Graphdiyne Nanowalls Using Acetylenic Coupling Reaction. J. Am. Chem. Soc. 2015, 137, 7596-7599.

(29)Bronner, C.; Marangoni, T.; Rizzo, D. J.; Durr, R. A.; Jørgensen, J. H.; Fischer, F. R.; Crommie, M. F. Iodine versus Bromine Functionalization for Bottom-Up Graphene Nanoribbon Growth: Role of Diffusion. J. Phys. Chem. C 2017, 121, 18490-18495.

(30) Tour, J. M. Top-Down versus Bottom-Up Fabrication of Graphene-Based Electronics. Chem. Mater. 2014, 26, 163-171.

(31)Chinchilla, R.; Nájera, C. Recent Advances in Sonogashira Reactions. Chem. Soc. Rev. 2011, 40, 5084-5121.

(32)Moreno, C.; Vilas-Valera, M.; Kretz, B.; Garcia-Lekue, A.; Costache, M. V.; Paradinas, M.; Panighel, M.; Ceballos, G.; Valenzuela, S. O.; Peña, D. et al. Bottom-Up Synthesis of Multifunctional Nanoporous Graphene. Science 2018, 360, 199-203.

(33) Ducéré, J. M.; Lepetit, C.; Chauvin, R. Carbo-Graphite: Structural, Mechanical, and Electronic Properties. J. Phys. Chem. C 2013, 117, 21671-21681.

(34) Lee, C.; Wei, X.; Kysar, J. W.; Hone, J. Measurement of the Elastic Properties and Intrinsic Strength of Monolayer Graphene. Science 2008, 321, 385-388.

(35) Yue, Q.; Chang, S.; Kang, J.; Qin, S.; Li, J. Mechanical and Electronic Properties of Graphyne and Its Family under Elastic Strain: Theoretical Predictions. J. Phys. Chem. C 2013, 117, 14804-14811.

(36) Kang, J.; Li, J.; Wu, F.; Li, S. S.; Xia, J. B. Elastic, Electronic and Optical Properties of Two-Dimensional Graphyne Sheet. J. Phys. Chem. C 2011, 115, 20466-20470. 
(37) Peng, Q.; Ji, W.; De, S. Mechanical Properties of Graphyne Monolayer: a FirstPrinciples Study. Phys. Chem. Chem. Phys. 2012, 14, 13385-13391.

(38) Cranford, S. W.; Buehler, M. J. Mechanical Properties of Graphyne. Carbon 2011, 49, 4111-4121.

(39) Sun, H.; Mukherjee, S.; Daly, M.; Krishnan, A.; Karigerasi, M. H.; Singh, C. V. New Insights into the Structure-Nonlinear Mechanical Property Relations for Graphene Allotropes. Carbon 2016, 110, 443-457.

(40) Nye, J. F. Physical Properties of Crystals; Oxford Science Publications: Oxford, U.K., 1985, pp. 134-135.

(41) Moon, H. S.; Yun, J. M.; Kim, K. H.; Jang, S. S.; Lee, S. G. Investigations of the Band Structures of Edge-Defect Zigzag Graphene Nanoribbons Using Density Functional Theory. RSC Adv. 2016, 6, 39587-39594. 